\title{
CONF-970911- -
}

Presented at Electron Microscopy and Analysis '97, Cambridge, United Kingdom, September 2-5, 1997; to be published in Proceedings

\section{DIRECT STRUCTURE DETERMINATION BY ATOMIC-RESOLUTION INCOHERENT STEM IMAGING}

P. D. Nellist,* Y. Xin, and S. J. Pennycook

*Cavendish Laboratory, University of Cambridge Madingley Road, Cambridge CB3 OHE, United Kingdom

Solid State Division, Oak Ridge National Laboratory

Oak Ridge, Tennessee 37831-6031

SOLID STATE DIVISION

OAK RIDGE NATIONAL LABORATORY Managed by

LOCKHEED MARTIN ENERGY RESEARCH CORP. under

Contract No. DE-AC05-96OR22464

with the

U.S. DEPARTMENT OF ENERGY

Oak Ridge, Tennessee

November 1997 


\section{DISCLAIMER}

This report was prepared as an account of work sponsored by an agency of the United States Government. Neither the United States Government nor any agency thereof, nor any of their employees, makes any warranty, express or implied, or assumes any legal liability or responsibility for the accuracy, completeness, or usefulness of any information, apparatus, product, or process disclosed, or represents that its use would not infringe privately owned rights. Reference herein to any specific commercial product, process, or service by trade name, trademark, manufacturer, or otherwise does not necessarily constitute or imply its endorsement, recommendation, or favoring by the United States Government or any agency thereof. The views and opinions of authors expressed herein do not necessarily state or reflect those of the United States Government or any agency thereof. 


\section{DISCLAIMER}

Portions of this document may be illegible electronic image products. Images are produced from the best available original document. 


\title{
Direct structure determination by atomic-resolution incoherent STEM imaging
}

\author{
P D Nellist, Y Xin* and S J Pennycook* \\ Cavendish Laboratory, University of Cambridge, Madingley Road., Cambridge CB3 0HE, \\ UK. \\ *Oak Ridge National Laboratory, Solid State Division, PO Box 2008, Oak Ridge, TN 37831- \\ 6031, USA.
}

\begin{abstract}
Use of a large, annular dark-field (ADF) detector in a scanning transmission electron microscope is shown to give images that can allow direct structure determination, being a convolution between the illuminating probe intensity and an object function localised at the atomic column positions. The $\mathrm{ADF}$ image is also shown to resolve crystal spacings more than twice smaller than the phase contrast point resolution limit of the microscope used, with sub-angstrom structural information being retrieved.
\end{abstract}

\section{INTRODUCTION}

In the determination of atomic structures by conventional high-resolution transmission electron microscopy (HRTEM), there are two important difficulties. First, because the electron interacts strongly with the electric potential within the crystal, strong multiple scattering occurs that can complicate the image intensity and in general renders it impossible to interpret the image intuitively in terms of the projected structure. Second, spherical aberration limits the angle of scattered electrons that can be focussed by the objective lens, giving a resolution limit that can limit the accuracy to which the positions of atomic can be determined, and can also give rise to artefacts in the image.

It is now more than 100 years since Lord Rayleigh (1896) considered the incoherent mode of imaging for light optics. He noted that illuminating the specimen with a large incoherent source renders the specimen effectively self-luminous, destroying interference effects between spatially separated parts of the specimen. In addition, he noted that the incoherent mode could resolve features with half the spacing of that for axial plane wave illumination. By the principle of reciprocity a large detector in a scanning transmission electron microscope (STEM) is equivalent to a large incoherent source in a conventional microscope. Because few electrons are absorbed in a thin specimen, an infinite detector that collected all transmitted electrons would give no contrast, so we add contrast by introducing a hole, giving an annular dark-field (ADF) detector (Fig. 1). An ADF image from a $\{1 \overline{2} 10\}$ domain boundary in wurtzite GaN viewed along $<0001>$ is shown in Fig. 2. The positions of the atomic columns are clear in this image and agree with the structure proposed by Xin et al. (1997). Here we describe briefly how such structure images arise, and how quantitative information may be retrieved from them.

\section{INCOHERENT IMAGING FROM COHERENT SCATTERING}

We start be considering the situation for a stationary lattice, so that the scattered electron wavefield can be assumed to be completely coherent, with purely Bragg diffraction for a crystalline specimen giving Bragg diffracted discs as depicted in Fig. 1. The Fourier transform of the ADF image intensity can be written in terms of the Fourier components of the 


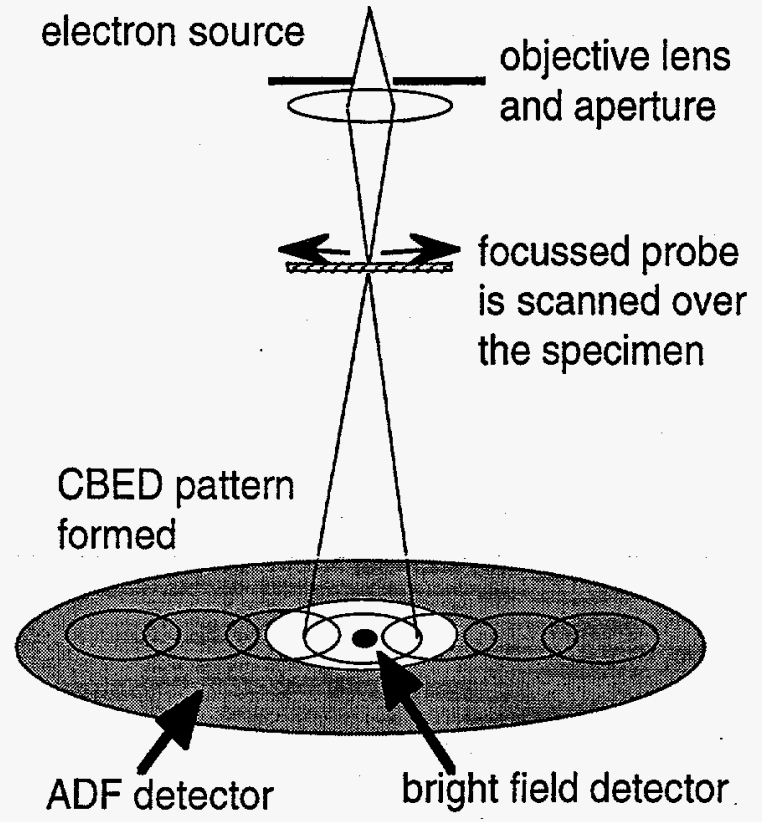

Fig. 1. A schematic of ADF image formation in a STEM

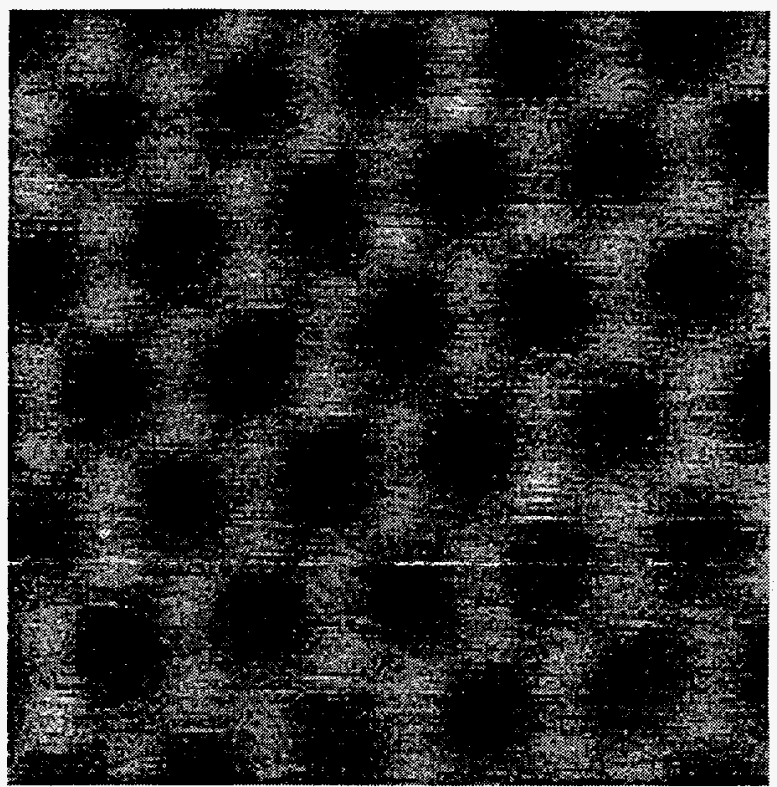

Fig. 2. A $\{1 \overline{2} 10\}$ domain boundary in wurtzite $\mathrm{GaN}$ viewed along $<0001>$. The atom columns are $0.19 \mathrm{~nm}$ apart.

Bloch wave solutions of the Schrödinger equation (Nellist and Pennycook, 1997a). The advantage of such a formulation is that it is possible to perform the summation over the Bragg beams incident on the ADF detector before summing over the Bloch waves. It is then possible to determine which Bloch states contribute to the ADF image. A calculation for GaAs viewed along $<110\rangle$ at $300 \mathrm{kV}$ shows that for a $30 \mathrm{mrad}$ inner detector radius, the image contrast is dominated by the contribution from the tightly bound 1s-type states (Fig. 3). It is only the 1s states that have the sufficient transverse momentum to scatter the electrons to the detector. Even if we now include the effect of lattice vibrations, phonon scattering is not able to provide the high-transverse momentum required, and only helps to destroy interference effects between the high-angle elastic scattering from different heights within a single column. We can therefore think of the ADF detector as a Bloch state filter.

Since the excitation and form of the 1s-type states, which dominate the ADF image contrast, vary only slowly as a function of the incident beam angle, all the coherent partial plane waves in the probe-forming incident cone of illumination can contribute with similar weight. In this 
situation it is straightforward to show (Nellist and Pennycook, 1997b) that the image can be treated as a convolution between an object function formed from the highly localised 1s type states and the probe intensity,

$$
I(\mathbf{R})=\int\left|P\left(\mathbf{R}^{\prime}-\mathbf{R}\right)\right|^{2} O\left(\mathbf{R}^{\prime}\right) d \mathbf{R}^{\prime}
$$

where $P(\mathbf{R})$ is the complex amplitude of the illuminating probe, and $O(\mathbf{R})$ is the object function. Since the intensity of the probe is a real and positive quantity, it can be immediately deconvolved from the image intensity, in contrast to the coherent imaging process where in general it is necessary to solve for the phase of the image-plane before a deconvolution can be performed.

\section{DECONVOLUTION AND RESOLUTION}

Equation (1) can be used in two ways: If the probe intensity function is known, then it can be deconvolved to give information on the object function that allows greater accuracy in structure determination. This is useful because in any resolution limited imaging system, the peak intensity positions will not necessarily be located at the atomic column positions. Alternatively, if the object function is known, which often happens in practice because a typical image will contain a defect surrounded by regions of perfect crystalline matrix, then it is possible to find an estimate for the probe intensity (Nellist and Pennycook, 1997b). Having determined the probe intensity, we need to consider how it may be deconvolved from the experimental image intensity. Fig. 4 shows raw data from an image of GaAs $<110\rangle$ and the result when the probe intensity function is deconvolved from it using a Wiener filter. Although the deconvolution has reduced the noise level in the image, the resulting object function is not localised, and there are stronger artefacts in the tunnels between the dumbbells. The reason that an object function with localised peaks at the atoms columns has not been reconstructed is because the deconvolution has not been able to reconstruct information beyond the resolution limit of the microscope. There is not one unique object function that fits the data, and to find the atomic locations accurately a further constraint is required (Nellist and Pennycook, 1997).

For the accurate determination of structures, therefore, the achievement of higher resolution in TEM is an important goal, even when the projected interatomic spacings are within the resolution limit of the microscope. For the same imaging conditions, the ADF image is able to resolve spacings half as small as conventional bright-field imaging, as indicated in Fig. 1 where the bright-field image would not show any contrast. For ADF imaging, using a large objective aperture with a highly underfocussed lens can pass information well beyond the conventional Scherzer incoherent resolution limit, albeit with some loss in the intuitive nature of the image. Fig. 5 shows the magnitude of the Fourier

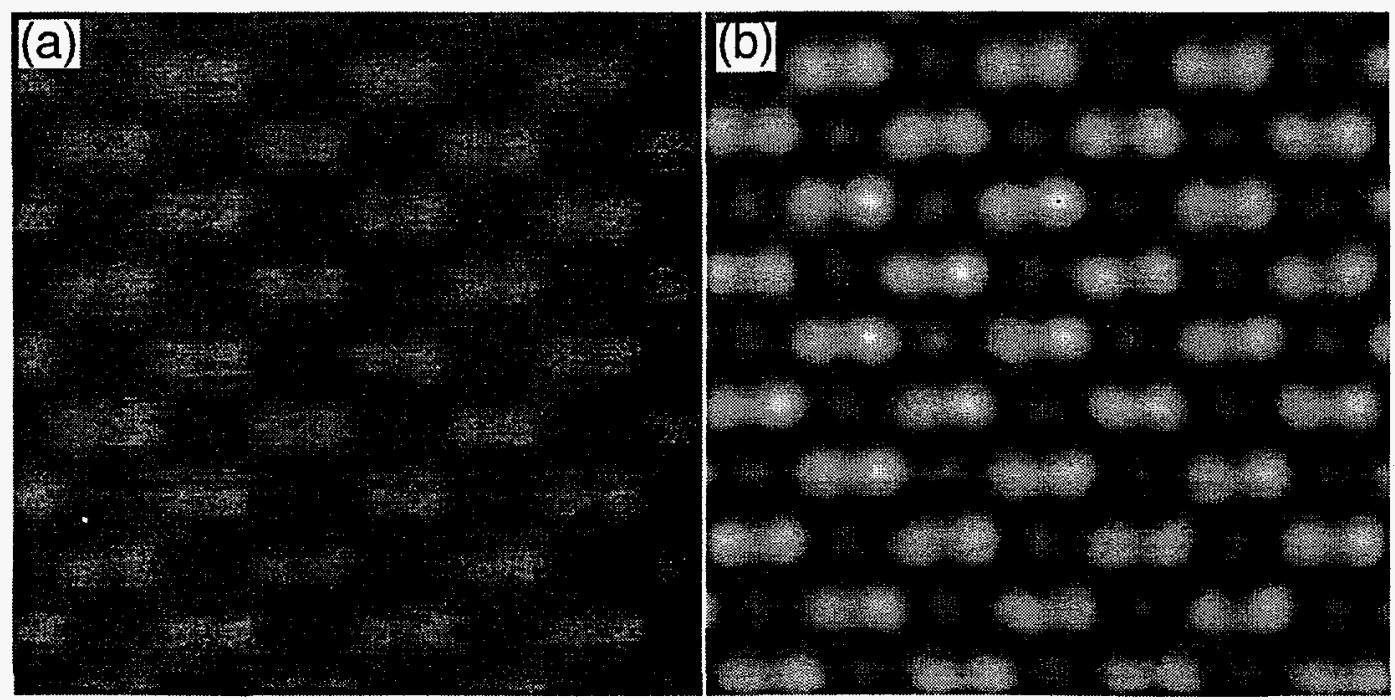

Fig. 4. (a) Raw image data of $\mathrm{GaAs}<110>$ and (b) the image when the probe intensity function has been deconvolved using a Wiener filter. 
transform of an image of $\mathrm{Si}<110>$ taken using an objective aperture $180 \%$ larger than the optimum Scherzer size, and with the objective lens approximately $130 \mathrm{~nm}$ underfocussed. A (444) spatial frequency may be seen, which indicates information transfer to $0.078 \mathrm{~nm}$. Data taken from a sample of $\mathrm{CdTe}<11 \overline{2}>$ under similar conditions is shown in Fig. 6. A (444) spatial frequency can be seen in both the image intensity profile and the magnitude of the Fourier transform, thus resolving the $0.093 \mathrm{~nm}$ spacing between the $\mathrm{Cd}$ and Te columns in this projection. The phase relationship between the (111) and (444) image spatial frequencies should allow the positions and polarity of the $\mathrm{Cd}$ and $\mathrm{Te}$ atomic columns to be determined.

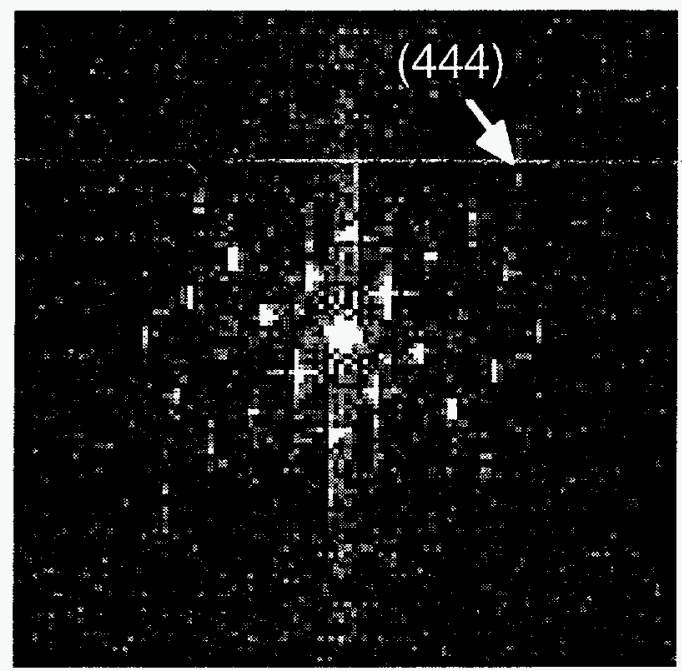

Fig. 5. The magnitude of the Fourier transform of a highly undefocussed ADF image of $\mathrm{Si}<110>$ using an oversized objective aperture.

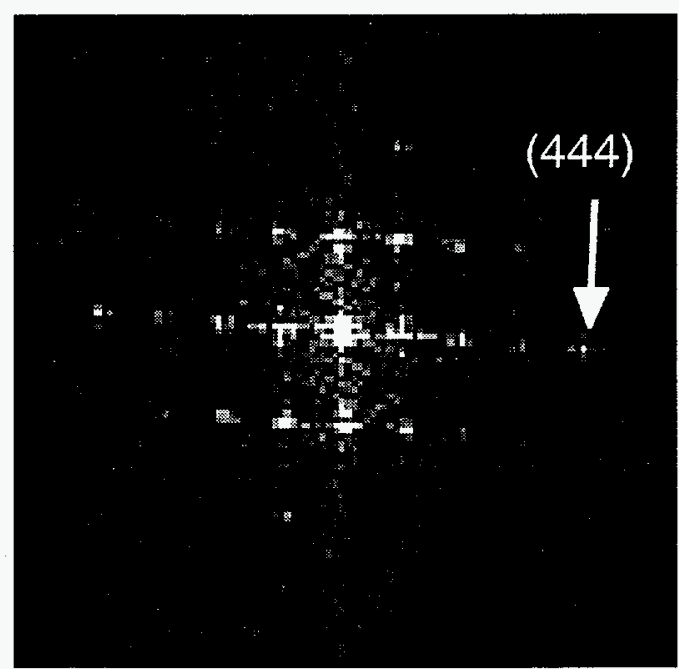

$0.093 \mathrm{~nm}$ spacing resolved, and showing polarity information

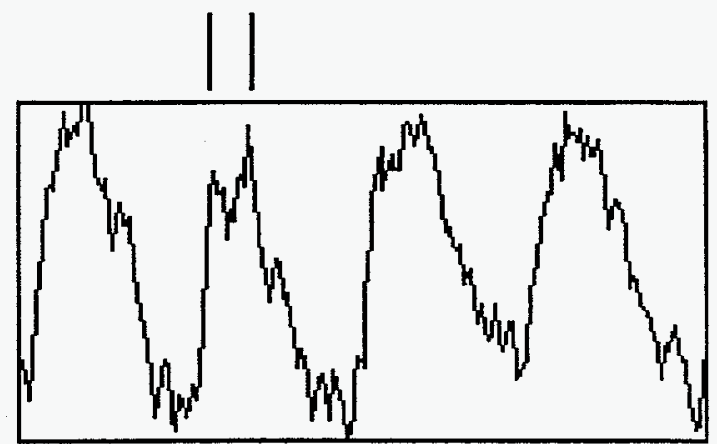

Fig. 6. The magnitude of the Fourier transform of an image of $\mathrm{CdTe}<11 \overline{2}>$ taken using similar conditions to Fig. 5 along with an image profile along the [111] direction..

Lord Rayleigh 1896 Phil. Mag. (5) 42, 167

Xin Y, Brown P D, Humphreys C J, Cheng T S and Foxon C T (1997) Appl. Phys. Lett. 70, 1308

Nellist P D and Pennycook S J (1997a) to be published

Nellist P D and Pennycook S J (1997b) J. Microsc. in press

This research was sponsored by the US DOE under contract no. DE-AC05-84OR21400 with LMER corp. and by a Post-doctoral appointment to ORISE. 